\title{
Spectroscopic monitoring of bright A-F type candidate hybrid stars discovered by the Kepler mission*
}

\author{
Patricia Lampens ${ }^{1}$, Yves Frémat ${ }^{1}$, Lore Vermeylen ${ }^{1}$, Peter De Cat ${ }^{1}$, \\ Louis Dumortier $^{1}$, Ádám Sódor ${ }^{2}$, Marek Skarka $^{2}$, Zsófia Bognár $^{2}$ \\ ${ }^{1}$ Royal Observatory of Belgium, Ringlaan 3, B-1180 Brussels, Belgium \\ ${ }^{2}$ Konkoly Observatory, MTA CSFK, Konkoly Thege M. u. 15-17, H-1121 Budapest, Hungary
}

\begin{abstract}
We report on a study of 250 optical spectra for 50 bright A/F-type candidate hybrid pulsating stars from the Kepler field. Most of the spectra have been collected with the high-resolution spectrograph HERMES attached to the Mercator telescope, La Palma. We determined the radial velocities (RVs), projected rotational velocities, fundamental atmospheric parameters and provide a classification based on the appearance of the cross-correlation profiles and the behaviour of the RVs with time in order to find true hybrid pulsators. Additionally, we also detected new spectroscopic binary and multiple systems in our sample and determined the fraction of spectroscopic systems. In order to be able to extend this investigation to the fainter A-F type candidate hybrid stars, various high-quality spectra collected with 3-4 m sized telescopes suitably equipped with a high-resolution spectrograph and furthermore located in the Northern hemisphere would be ideal. This programme could be done using the new instruments installed at the Devasthal Observatory.
\end{abstract}

\section{Introduction}

The goal of the NASA space mission Kepler (2009-2014) is to search for transiting exoplanets by continuously monitoring some 200,000 main-sequence and giant stars in the Kepler field (Borucki et al. 2010). Such continuous flux measurements of unprecedented photometric precision are also extremely useful for studies of different kinds of stellar variability (including pulsation, rotation, activity and multiplicity). For example, these space-based observations lead to a catalogue of 2878 eclipsing binary systems discovered in the entire Kepler field which is recently published (Kirk et al. 2016).

Among the main-sequence stars with a mass between 1.5 and 2.5 solar masses, two very different classes of pulsating stars exist: these are the $\gamma$ Dor stars (with gravity-driven modes and frequencies in the range $0-5 \mathrm{c} / \mathrm{d}$ ) and the (slightly) hotter $\delta$ Scuti stars (with pressure-driven modes and frequencies in the range 5-80 c/d). Theory tells us that, when such stars have a surface temperature between 6900 and $7400 \mathrm{~K}$, they can have both types of pulsation modes excited and are then called "hybrid"

${ }^{*}$ Based on observations obtained with the Hermes spectrograph, which is supported by the Research Foundation Flanders (FWO), Belgium, the Research Council of KU Leuven, Belgium, the Fonds National de la Recherche Scientifique (F.R.S.-FNRS), Belgium, the Royal Observatory of Belgium, the Observatoire de Genève, Switzerland and the Thüringer Landessternwarte Tautenburg, Germany. 
stars. These hybrid stars undergo light variations in both regimes of frequencies. The frequency limit between the two regimes is arbitrarily set to $5 \mathrm{c} / \mathrm{d}$. The space missions CoRoT and Kepler provided a wealth of such candidate hybrid stars, many of which are located outside the theoretically predicted physical parameter range (Grigahcène et al. 2010, Uytterhoeven et al. 2011, Hareter 2012). This discovery represents a major challenge for the theory of stellar pulsation (Dupret et al. 2005, Balona et al. 2015). Such objects are powerful asteroseismic targets since they provide a complete description of the stellar interiors, i. e. from the inner cores to the outer envelopes.

In our study, we address the question whether all these objects are true hybrid pulsators, i.e. whether they undergo both low-order pressure- (modes 'p') and high-order gravity-modes (modes ' $g$ '). Various physical causes can indeed explain the presence of low frequencies in the periodograms of these stars:

- Confirmed hybrid pulsation (i.e. co-existence of modes 'p' and 'g'). However, Balona (2014) noted that it is not understood how low frequencies can be driven in the absence of a substantial convective zone. Stars hotter than $T_{\mathrm{eff}} \approx 7500 \mathrm{~K}$ do not have a sufficiently deep convective zone for the convective blocking mechanism to operate.

- Rotational modulation. The rotation of an inhomogeneous stellar surface, e.g. due to chemical anomalies as in Am/Ap stars or, as a consequence of a strong temperature gradient in a fast rotating star (as Altair), may introduce flux variations with frequencies which are coupled to the stellar rotation. It seems that rotation plays an important role in the appearance of the low-frequency peaks of normal A-type stars of the Kepler mission (Balona 2011).

- Undetected binarity. The components of ellipsoidal or eclipsing binaries may have distorted shapes caused by tides. The binary systems may present grazing/shallow eclipses, or we may have the case of a binary with a $\gamma$ Dor and a $\delta$ Scuti companion. We may also think of close binary systems in an eccentric orbit in which tidally-driven gravity modes can appear (e.g. Hambleton et al. 2013). In such a system, the excitation of the 'g' modes is forced by the dynamic tides.

The confirmation of genuine cases of hybrid pulsators among the candidate stars thus represents an important step in the study of the new A/F-type hybrid phenomenon.

\section{Observations}

A large amount of spectra was acquired with the high-resolution fibre-fed échelle spectrograph HERMES (High Efficiency and Resolution Mercator Echelle Spectrograph (Raskin et al. 2011) equipping the 1.2-m Mercator telescope situated at the international observatory Roque de los Muchachos (La Palma, Spain). The instrument is operated by the University of Leuven under the supervision of the HERMES Consortium and in use since 2009. It records the optical spectrum in the range $\lambda=377$ $900 \mathrm{~nm}$ across 55 spectral orders in a single exposure. The resolving power in the high-resolution mode (optimised for high efficiency observations) is $\mathrm{R}=85000$. A dedicated pipeline ensures automatic reduction of these échelle spectra but without normalization. The normalization is performed afterwards and independently by the researcher.

We also collected additional spectra with the recent ACE fibre-fed échelle spectrograph attached to the 1-m Ritchey-Chrétien telescope of the Konkoly Observatory located at Piszkés-tetô, Hungary. 


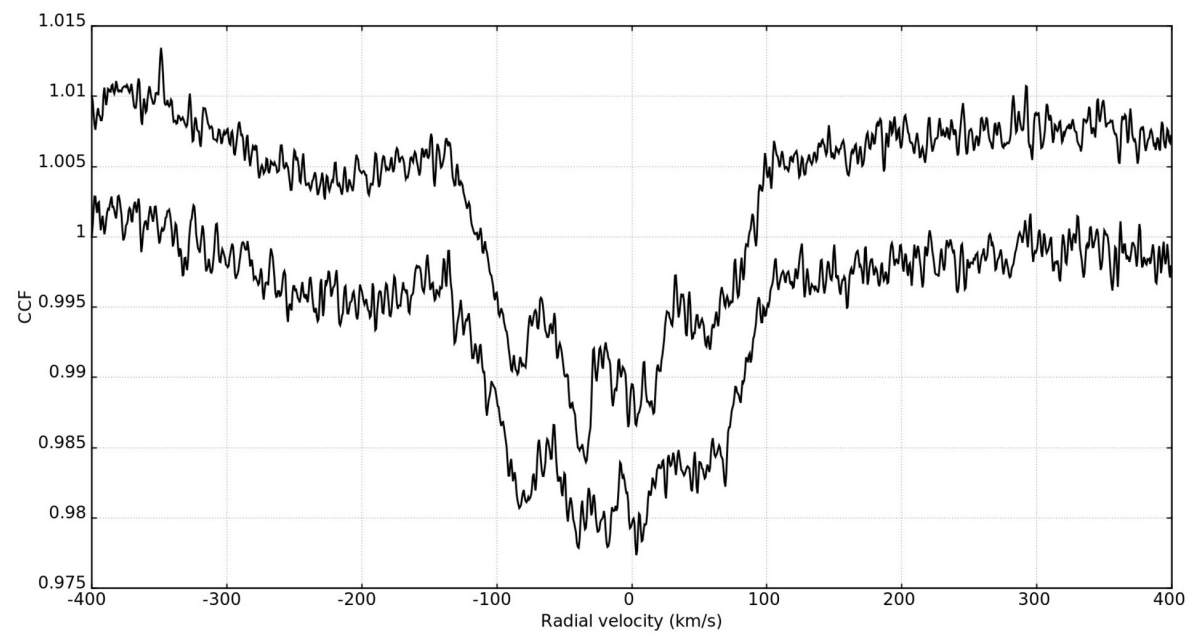

Figure 1: These are two 1-D CC functions of KIC 3437940.

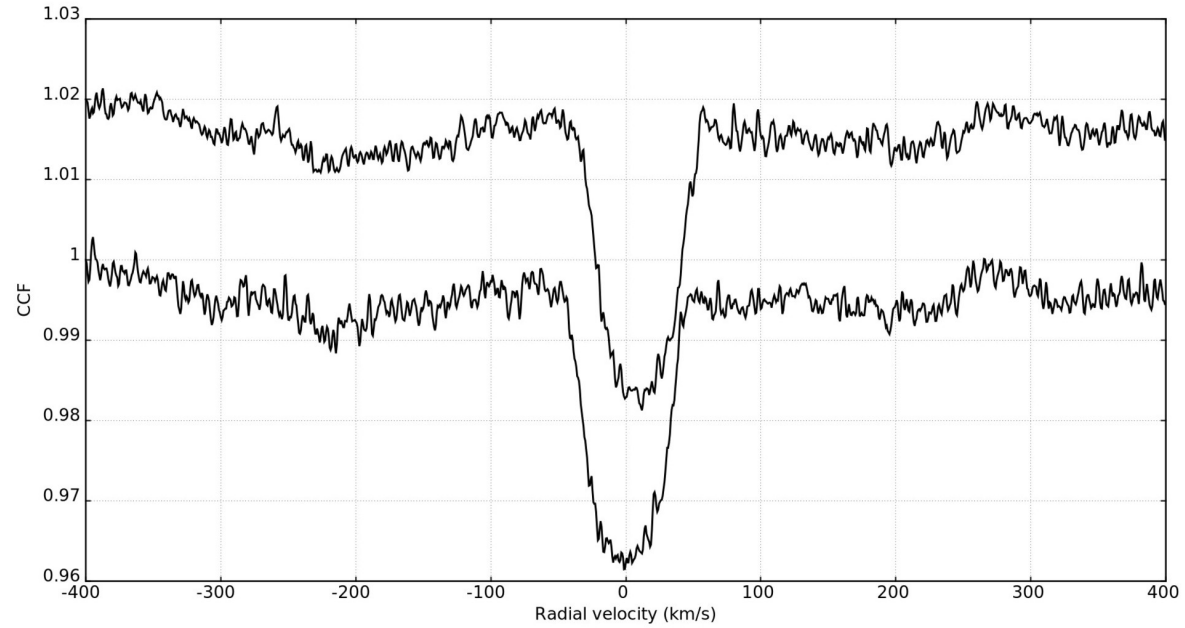

Figure 2: These are two 1-D CC functions of KIC 9790479.

The ACE spectrograph covers the $415-915 \mathrm{~nm}$ wavelength range with a resolution $\mathrm{R}=20000$. More information on this spectrograph, which is in use since 2014, can be found in Derekas et al. (2016).

\section{Spectroscopic analyses}

\subsection{Cross-correlation functions and radial velocities}

We defined up to 10-11 (large) wavelength intervals per spectrum and normalized each interval separately. First, we computed 1-D cross-correlation (CC) functions in order to find a suitable set of model parameters in terms of the number of components, spectral type(s) and $v \sin i$ for each target. The radial velocities were obtained by fitting the model to the observed part(s) of the spectrum. By repeating this step at least ten times, we obtained the mean radial velocity and its standard deviation.

For illustration, we present two CC profiles of KIC 3437940 (Fig. 1). For the model we used (sp. type A7, $v \sin i=110 \mathrm{~km} \mathrm{~s}^{-1}$ ). Note that the shape of the CC profile shows the presence of 


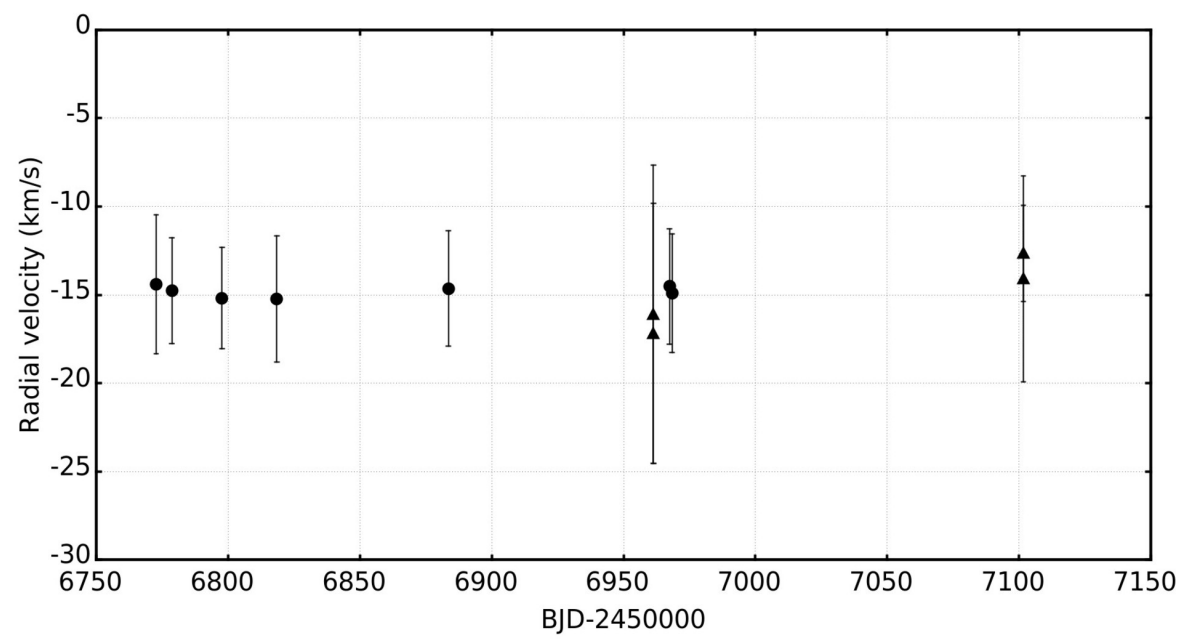

Figure 3: RV curve of KIC 3437940.

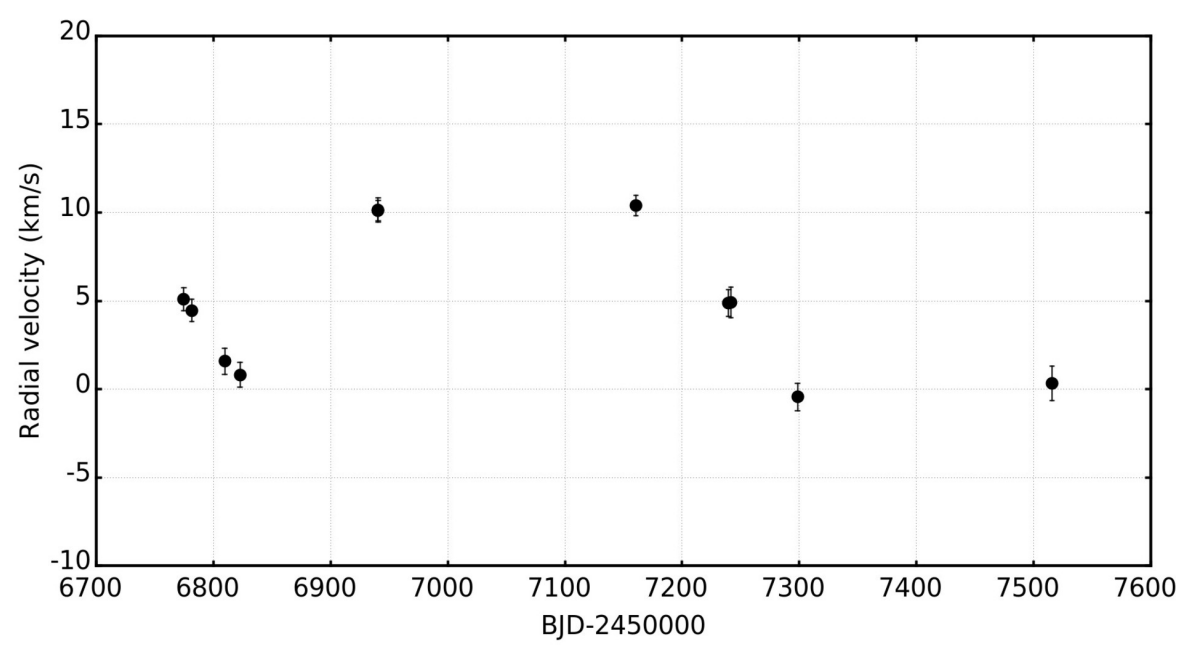

Figure 4: RV curve of KIC 9790479.

moving bumps, which is the possible signature of non-radial pulsation. The corresponding radial velocity curve over time appears to be constant (Fig. 3). We classified this target as a single star and 'P(ulsating)'.

As a second illustration, we present two CC profiles of KIC 9790479 (Fig. 2). For the model we used (sp. type A5, $v \sin i=40 \mathrm{~km} \mathrm{~s}^{-1}$ ). The profiles are stable in shape but shifted in velocity. The corresponding radial velocity curve over time appears to be variable (Fig. 4). We classified this target as a single-lined system, i. e. 'SB1'.

In the case of a composite spectrum (detected in the 1-D CC functions), several 2-D CC functions are computed for each wavelength interval. This procedure serves to derive approximate parameters of the composite model spectrum (in terms of spectral types and $v \sin i$ ) and to determine the corresponding component radial velocities. With these input parameters, we then construct a rotationally broadened, Doppler-shifted composite model spectrum which we try to match to the observed spectrum by minimizing the residual sum of squares as a function of the unknown light ratio, $\alpha$. We thus explore the parameter space in terms of spectral types and $v \sin i$. The most consistent and best-fitting model is retained and used for the computation of the final component radial velocities. 


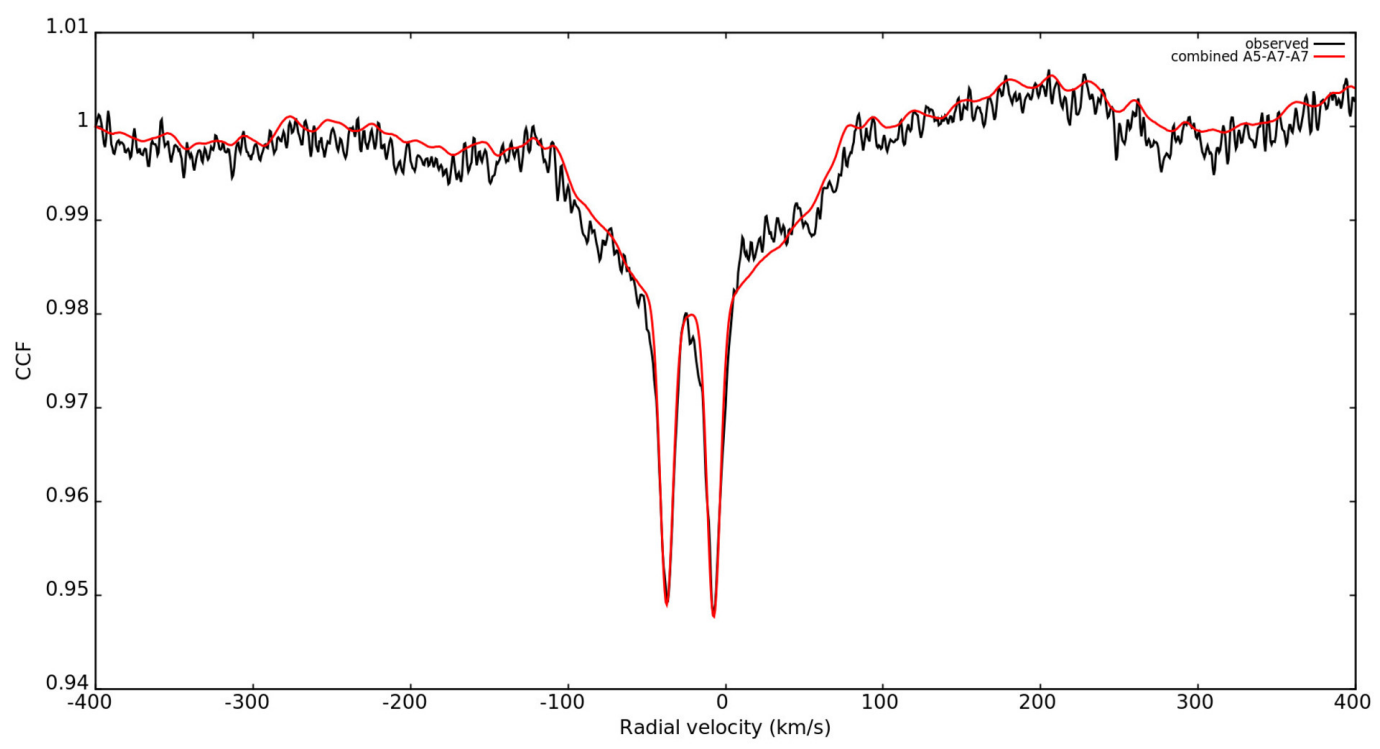

Figure 5: This is the 1-D CC function of the new triple system KIC 6381306 (in black: the data, in red: the model with three components).

We next present the case of KIC 6381306 as an illustration of a composite object. We discovered that the 1-D CC profiles are variable and show one broad and two deep minima. It is best matched with a model consisting of one component of type (A5, $\left.v \sin i=90 \mathrm{~km} \mathrm{~s}^{-1}\right)$ and a twin system with two components of type (A7, $v \sin i=5 \mathrm{~km} \mathrm{~s}^{-1}$ ) (Fig. 5). Based on the phase-distributed (component) radial velocities, we determined that the inner binary moves in a short-period orbit with the following orbital elements: period $=3.9$ days, eccentricity $=0$, and RV amplitudes $\mathrm{K}_{1}=\mathrm{K}_{2}=19 \mathrm{~km} \mathrm{~s}^{-1}$ (Fig. 6). We thus classified this target as a triple-lined system, i. e. 'SB3'.

\subsection{Fundamental parameters}

In order to determine improved fundamental atmospheric parameters, we confronted the observed spectra with various models using the building code SYNSPEC (Hubeny \& Lanz 1995) together with the ATLAS9 model atmospheres (Castelli \& Kurucz 2003). The comparison between the observations and a grid of models and the determination of the final set of parameters is performed with the code GIRFIT (Frémat et al. 2006). In the case of KIC 6432054 (classified as stable and 'P(ulsating)'), we used (sp. type A7, $v \sin i=180 \mathrm{~km} \mathrm{~s}^{-1}$ ) as a start. From the grid exploration, we obtained the following properties: $T_{\text {eff }}=7540 \mathrm{~K}, \log g=4.4, v \sin i=184 \mathrm{~km} \mathrm{~s}^{-1}$. In Fig. 7, we show the good agreement between the observed spectrum and the model with the above mentioned parameters.

\section{Incidence of binarity and multiplicity}

Our sample of 50 targets consists of the brighter A- and F-type candidate hybrid stars of the $\mathrm{Ke}$ pler mission first explored by Uytterhoeven et al. (2011). Based on a large (but incomplete) number of HERMES and ACE spectra, we classified all our targets into five distinct categories: Stable (S), 


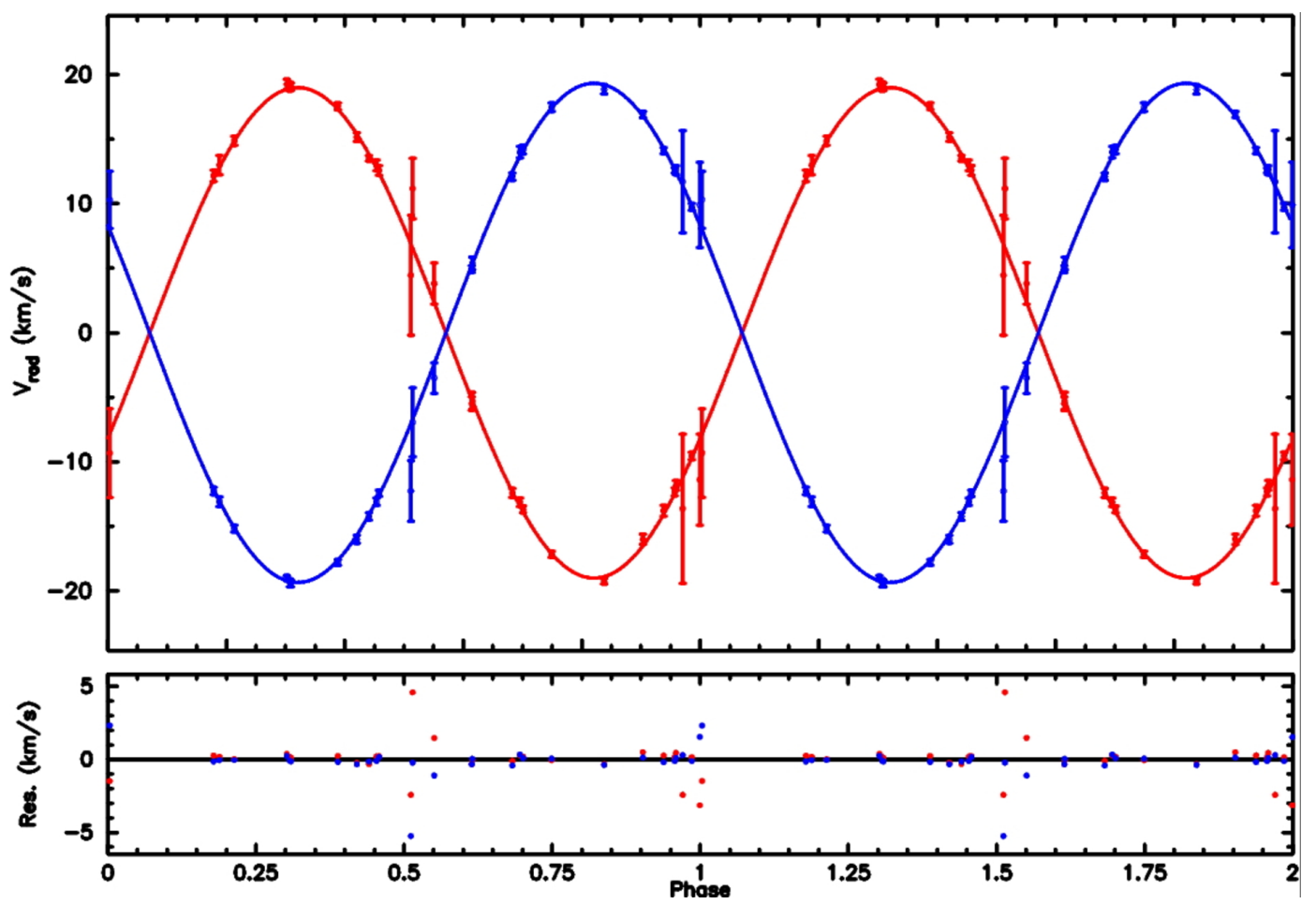

Figure 6: This plot illustrates the orbital solution for the inner binary system of KIC 6381306.

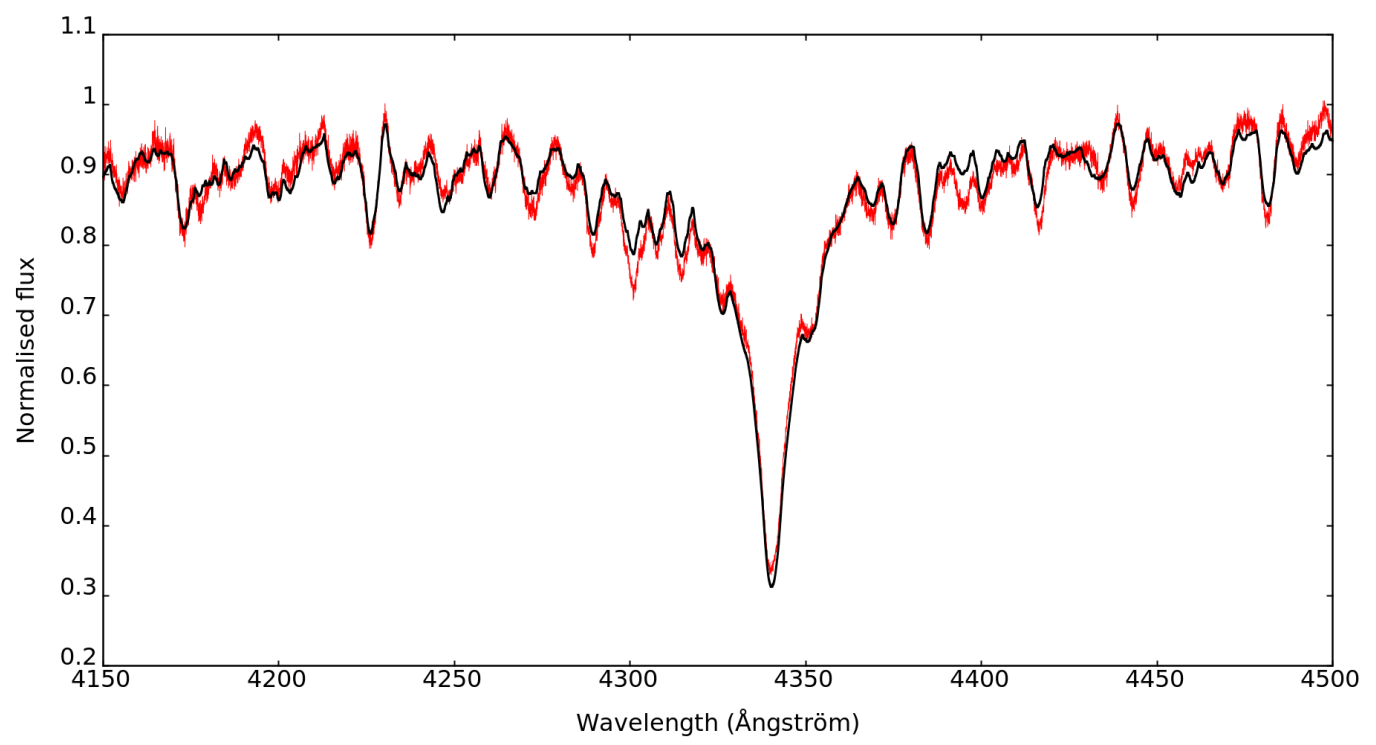

Figure 7: Part of the observed spectrum for KIC6432054 (in black) and model (in red) using the best-fit set of parameters obtained with the code GIRFIT. 
Pulsation or Rotation (P), Double or Multiple system (D\&M), long-term RV variable (VAR), and Composite spectrum (CMP). From this classification, we obtained the following distribution: $47 \%$ (S), 22\% (P), 20\% (D\&M), 6\% (VAR) and 4\% (CMP). Since both the long-term RV variable and the composite-spectrum objects may turn out to be (most probably SB1) systems on the long run, we conclude that the global multiplicity fraction in our sample of candidate hybrid pulsators is $\approx 30 \%$.

\section{$5 \quad$ Time delay analysis}

Since all our targets have accurate, continuous and multi-year Kepler photometry and undergo pulsations of the $\delta$ Scuti-type, we additionally searched for frequency modulations in the high-frequency regime possibly induced by orbital motions. This is the Light Travel Time effect (LTT). We computed the time delays for the most dominant highest frequencies of the stars from our sample. These results indicate consistent, long-term variability of the time delays in nine cases, six of which were already identified as new systems in the spectroscopic analyses.

\section{Conclusions \& perspectives}

Our study offers complementary spectroscopic data for the newly discovered candidate hybrid pulsators of the Kepler mission. We attest, based on this work, that high-resolution spectroscopy is highly complementary to space photometric data. The analysis of the CC profiles and the behaviour of the radial velocities with time leads to the orbital characterization of the new systems and provides a (minimal) estimation of the incidence of spectroscopic multiplicity in the sample. The confrontation of the observed spectra with models furthermore leads to the determination of improved fundamental atmospheric properties of the selected targets. Full results will appear in a future publication (Lampens et al., submitted to A\&A).

In the future, we would like to extend this type of study to a larger sample of fainter candidate hybrid stars of the Kepler mission. High-resolution spectra are also needed for in-depth studies of selected candidate hybrid stars, e.g. time-series of line-profile variations in various genuine hybrid stars, as well as for deriving strict constraints on the pulsational properties for the binary systems with pulsating components based on an accurate model of the system geometry. In the very important case of eclipsing systems with pulsating components, fast multi-colour photometry during the eclipses can procure significant insight for mode identification purposes (using the eclipse phenomenon as a spatial filter to resolve the pulsations across the stellar surface of the pulsator, e. g. Gamarova et al. (2002)). Finally, there is also a need for spectropolarimetry in order to check for the presence of brightness or chemical inhomogeneities on the surfaces of the candidate hybrid stars which may be related to magnetic activity (e. g. Neiner \& Lampens 2015). For such detailed stellar physics problems, the 4-m sized Devasthal Optical Telescope (DOT) equipped with suitable instrumentation is a very promising instrument. 


\section{Acknowledgements}

The authors are most grateful to the Federal Science Policy of Belgium (BELSPO) for funding of the BINA (Belgo-Indian Network for Astronomy \& Astrophysics) project. This research is based on highresolution spectra obtained with the HERMES échelle spectrograph installed at the Mercator telescope, operated by the IvS, KULeuven, funded by the Flemish Community, and located at the Observatorio del Roque de los Muchachos, La Palma, Spain, of the Instituto de Astrofisica de Canarias, as well as with the ACE échelle spectrograph attached to the 1-m RCC telescope of the Konkoly Observatory at Piszkés-tetô, Hungary. M.S. acknowledges the support of the postdoctoral fellowship programme of the Hungarian Academy of Sciences. Á.S., M.S. and Zs.B. acknowledge the financial support of the Hungarian NKFIH Grants K-115709 and K-119517. Á.S. was also supported by the János Bolyai Research Scholarship of the Hungarian Academy of Sciences.

\section{References}

Balona L. A. 2011, MNRAS, 415, 1691

Balona L. A. 2014, MNRAS, 437, 1476

Balona L. A., Daszyńska-Daszkiewicz J., Pamyatnykh A. 2015, MNRAS, 452, 3073

Borucki W., Koch D., Basri G. et al. 2010, Sci, 327, 5968, 977

Castelli F., Kurucz R.L. 2003, IAU Symposium, 210, A20

Derekas A., Plachy E., Molnár L. et al., 2017, MNRAS, 464, 1553

Dupret M.-A., Grigahcène A., Garrido R. et al., 2005, A\&A, 435, 927

Frémat Y., Neiner C., Hubert A.-M. et al., 2006, A\&A, 451, 1053

Gamarova A.Y., Mkrtichian D.E., Rodríguez E. et al. 2003, PASPC, 292, 369

Grigahcène A., Antoci V., Balona L. et al. 2010, ApJ 713, L192

Hambleton K. M., Kurtz D. W., Prša A. et al. 2013, MNRAS, 434, 925

Hareter M. 2012, AN, 333, 1048

Hubeny I., Lanz T. 1995, ApJ, 439, 875

Kirk B., Conroy K., Prša A. et al. 2016, AJ, 151, 68

Neiner C., Lampens P., 2015, MNRAS, 454, 86

Raskin G., Van Winckel H., Hensberge H. et al. 2011, A\&A, 526, A69

Uytterhoeven K., Moya A., Grigahcène A. et al. 2011, A\&A, 534, A125 